\title{
Beam and installation improvements of the NIO1 ion source
}

Cite as: Rev. Sci. Instrum. 91, 013316 (2020); https://doi.org/10.1063/1.5128658

Submitted: 20 September 2019 . Accepted: 18 December 2019 . Published Online: 16 January 2020

M. Cavenago (D), M. Barbisan, R. Delogu, A. Pimazzoni, C. Poggi, M. Ugoletti, M. Agostini, V. Antoni, C. Baltador, V. Cervaro, M. De Muri (D), D. Giora, P. Jain, B. Laterza, G. Maero (D), M. Maniero, D. Martini, A. Minarello, D. Ravarotto, D. Recchia, A. Rizzolo, M. Romé (D), E. Sartori, M. Sattin, C. Serianni (D), F. Taccogna (D), V. Valentino, V. Variale, and P. Veltri
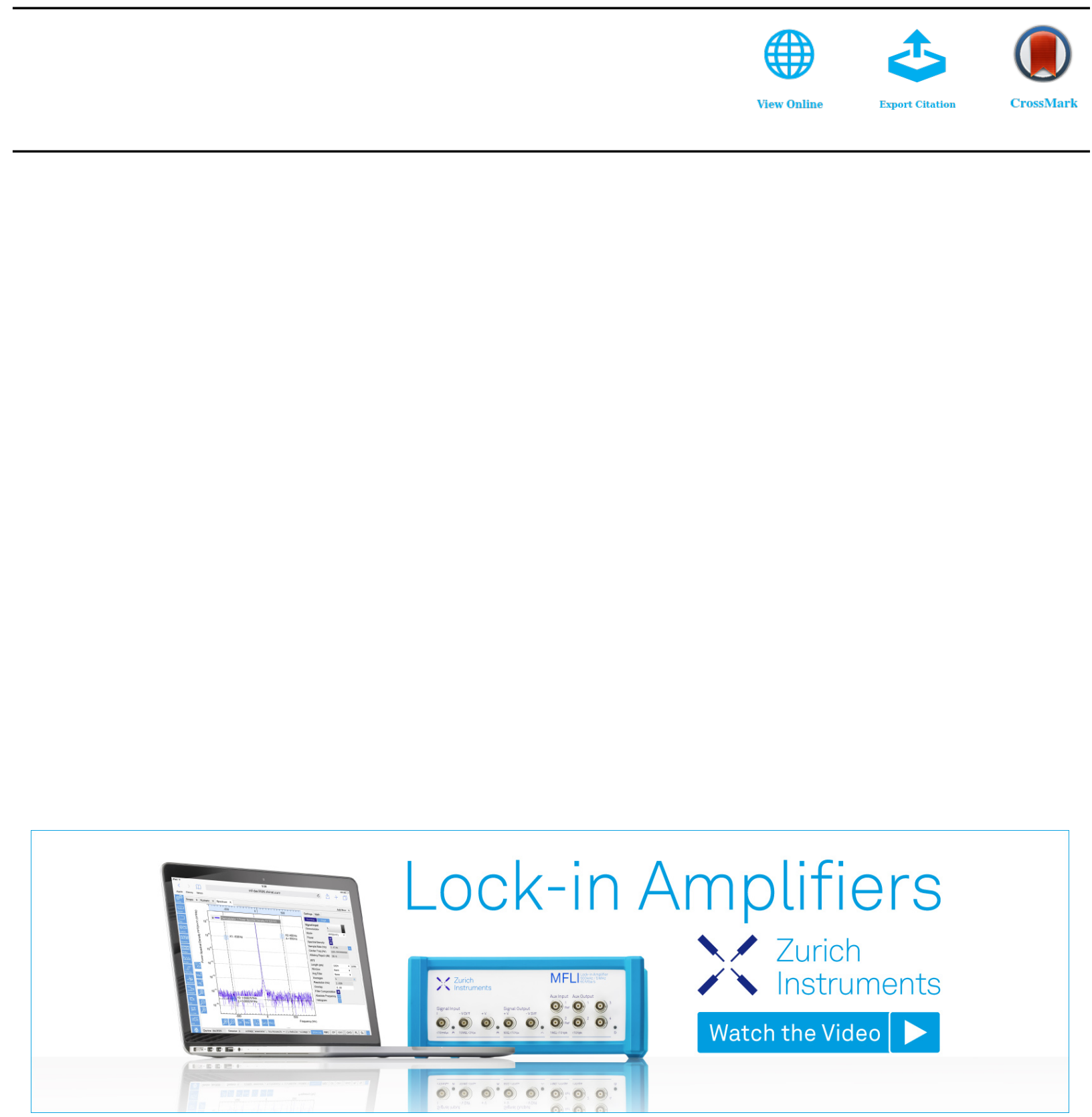


\title{
Beam and installation improvements of the NIO1 ion source
}

\author{
Cite as: Rev. Sci. Instrum. 91, 013316 (2020); doi: 10.1063/1.5128658 \\ Submitted: 20 September 2019 • Accepted: 18 December 2019 • \\ Published Online: 16 January 2020
}
M. Cavenago, ${ }^{1, a)}$ (iD M. Barbisan, ${ }^{2}$ R. Delogu, ${ }^{2}$ A. Pimazzoni,, C. Poggi, ${ }^{2}$ M. Ugoletti, ${ }^{2}$ M. Agostini, ${ }^{2}$ V. Antoni, ${ }^{3}$ C. Baltador,' V. Cervaro, ${ }^{2}$ M. De Muri, ${ }^{2}$ (D) D. Giora, ${ }^{1}$ P. Jain, ${ }^{2}$ B. Laterza, ${ }^{2}$ G. Maero, ${ }^{4}$ (D) M. Maniero, ${ }^{2}$ D. Martini, A. Minarello, ${ }^{7}$ D. Ravarotto, ${ }^{2}$ D. Recchia, ${ }^{2}$ A. Rizzolo, ${ }^{2}$ M. Romé, ${ }^{4}$ (D) E. Sartori, ${ }^{2}$ M. Sattin, ${ }^{1}$ G. Serianni, ${ }^{2}$ F. Taccogna, ${ }^{3}$ (D) V. Valentino, ${ }^{5}$ V. Variale, ${ }^{5}$ and P. Veltri ${ }^{6}$

\author{
AFFILIATIONS \\ ${ }^{1}$ INFN-LNL, v.le dell'Universitá 2, 35020 Legnaro (PD), Italy \\ ${ }^{2}$ Consorzio RFX, c. Stati Uniti 4, 35127 Padova, Italy \\ ${ }^{3}$ CNR-Istituto Scienza e Tecnologia dei Plasmi, c. Stati Uniti 4, 35127 Padova, Italy \\ «Univ. Milano and INFN-MI, v. Celoria 16, 20133 Milano, Italy \\ ${ }^{5}$ INFN-BA, v. Orabona 4, 70126 Bari, Italy \\ ${ }^{6}$ ITER-Organization, 13067 St. Paul Lez Durance Cedex, France
}

Note: Contributed paper, published as part of the Proceedings of the 18th International Conference on Ion Sources, Lanzhou, China, September 2019.

a) Author to whom correspondence should be addressed: cavenago@Inl.infn.it

\begin{abstract}
The NIO1 (Negative Ion Optimization phase 1) source can provide continuous beam operation, which is convenient for systematic parameter and equipment studies. Even in the pure volume production regime, the source yield was found to depend on conditioning procedures. Magnetic configuration tests continued adding magnets to the existing setup; the filter field component $B_{x}$ has been progressively extended to span the -12 to $5 \mathrm{mT}$ range, and as a trend, source performances improved with $\left|B_{x}\right|$. The progress of camera beam diagnostics and of the quality of the volume-produced $\mathrm{H}^{-}$beam is also shown. The status, off-line results, and reliability of a first NIO1 cesium oven are discussed; other upgrades in preparation (cavity ring down spectrometer, the end calorimeter, and conceptual tests of the energy recovery system) are
\end{abstract} also listed.

Published under license by AIP Publishing. https://doi.org/10.1063/1.5128658

\section{INTRODUCTION}

In view of the long term operation of neutral beam injectors (NBI) used for stellarator and tokamak heating and current drive, ${ }^{1,2}$ the negative ion source must be carefully optimized, ${ }^{3}$ especially because not only plasma but also surface wall conditions are involved in $\mathrm{H}^{-}$or $\mathrm{D}^{-}$production. The NIO1 source (Negative Ion Optimization phase 1 , described elsewhere ${ }^{4,5}$ ) was developed and has been operating since 2014 (in close collaboration between Consorzio RFX and INFN), as a convenient benchmark to study innovative solutions and to address physical questions. The comparatively smaller size of NIO1 (a compact 9 beamlet $\mathrm{H}^{-}$source) is advantageous for experimental changes, whose significance is amplified by the fact that NIO1 works in the Continuous Wave (CW) regime, as is necessary for DEMO.

As a first example of changes, several configurations (setups) of the source magnetic filter $\mathbf{B}^{s}$ were investigated (see Ref. 5); here, we add results obtained with the new setups " $\mathrm{f} 2$ " and " $\mathrm{f} 3$ " (described later). As another example, relations between source conditioning, evolution of $\mathrm{H}^{-}$output, and radio frequency (rf) window cooling were noted and investigated. The paper is organized as follows: first, the overall plan of NIO1 is reviewed, describing a new beam camera diagnostics and remarkable conditioning procedures. Preparation of planned upgrades (cesium, cavity ring down, and calorimeter) is also reported. Second, the new filter field is discussed, highlighting the recent improvement in beam current. 


\section{OVERALL NIOI PLAN, RESULTS AND UPGRADES}

The NIO1 plasma chamber (a $0.21 \mathrm{~m}$ long cylinder with $R_{c}=0.049 \mathrm{~m}$ radius, see Fig. 1) has 9 extraction apertures (arranged in 3 rows and 3 columns with $L_{x}=0.014 \mathrm{~m}$ spacing), each with $R_{h}=3.8 \mathrm{~mm}$ radius, in the so-called plasma grid (PG), which is connected to a high voltage deck (HVD), held to potential $-V_{s}$ with respect to earth. The accelerator consists of 3 electrodes, namely, the extraction grid (EG) held to potential $V_{e}$ with respect to the HVD and PG, the post acceleration (PA) grid connected to earth through an ammeter or a low voltage power supply, the repeller electrode (REP), followed by a $2-\mathrm{m}$ long drift section made out of a pumping cross, a diagnostic tube, and an end flange (see Fig. 2), where a calorimeter with pepper pot holes is to be placed.

Strong permanent magnets (PMs) are embedded in the EG and the PA, giving a field $B^{d}$ to deflect electrons out of the acceleration direction, which is our $z$ axis. Let the $x$ axis be aligned with these magnet longer sides; ${ }^{6}$ let $z=0$ be the PG plane so that the source plasma region is $z<0$ and the accelerator one is $z>0$. Then, $B^{d}$ is roughly aligned with the $y$-axis and negligible for $z \ll 0$. Let $p_{v}$ be the pressure measured in the diagnostic tube and $p_{s}$ the one in the source, the latter corrected for $\mathrm{H}_{2}$ gas.

Up to now, the beam is intercepted by a fixed Carbon Fiber Composite (CFC) tile, similar to the spectral shear interferometry for direct electric field reconstruction (SPIDER) calorimeter, and biased to a voltage $V_{\text {cfc }}$ in order to suppress secondary electron emission, hence collecting a nominal current signal $I_{\mathrm{cfc}}$. The effect of $V_{\text {cfc }}$ on $I_{\text {cfc }}$ is well-known; at lower $V_{\text {cfc }}<30 \mathrm{~V}$, the $\mathrm{H}^{-}$current is canceled by the electrons escaping the CFC, and at higher $V_{\text {cfc }}$, these $\mathrm{e}^{-}$are attracted back to the CFC. However, the measurement is disturbed by the capture of other $\mathrm{e}^{-}$produced by beam-gas collisions; up to now, we set $V_{\text {cfc }} \cong 60 \mathrm{~V}$ as a compromise between these errors. We plan to transform this tile into a removable Faraday Cup (FC). A comparison with calorimetric CFC measurements of the beam current $I_{\text {cal }}$ and power supply net current $I_{s}^{n}$ (subtracting current into known resistances measured with plasma off) was done only in a few experiments, mostly at $V_{s}=10.4 \mathrm{kV}, V_{s} / V_{e}=8$, depending on the infrared camera

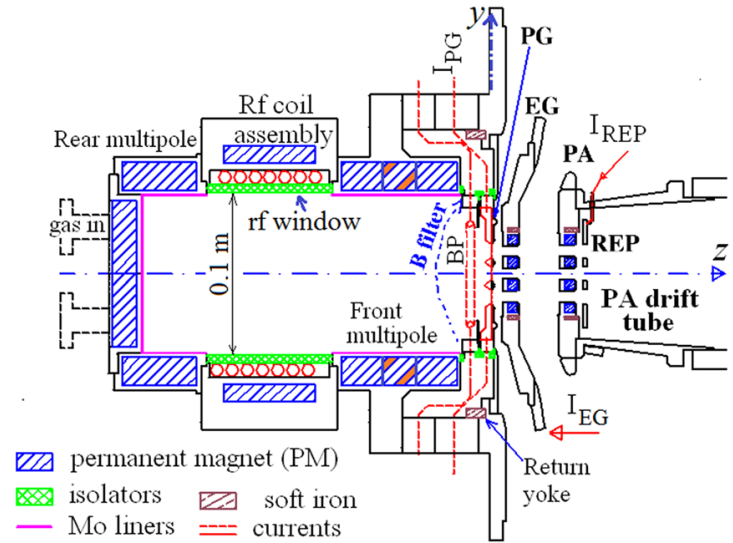

FIG. 1. Section zy of the NIO1 source with plasma grid PG and post extraction grid PA (see also the text).

availability; for a typical example, with $p_{s}=0.75 \mathrm{~Pa}, p_{v}=0.02 \mathrm{~Pa}$, and $I_{\mathrm{cfc}}=2.3 \mathrm{~mA}$, we got $I_{\mathrm{cal}}=1.7 \mathrm{~mA}$ and $I_{s}^{n}=3.4 \mathrm{~mA}$.

Even if NIO1 has no Faraday shield, the rf power $P_{k}$ required to keep plasma on (level $P_{k 1}$ ) or to keep inductive coupling (level $P_{k 2}$ ) are similar in the case of hydrogen (say $P_{k 1} \cong 0.8 \mathrm{~kW}$ ) so that capacitive coupling is rarely observed, as opposed to the oxygen case. Without plasma, the $\mathrm{rf}$ coil resonant frequency is $2.01 \mathrm{MHz}$ with $140 \Omega$ impedance. With hydrogen plasma and $P_{k}>1 \mathrm{~kW}$, the rf can be set similarly (2.014 MHz mostly used) with no appreciable reflection for $p_{s}>0.6 \mathrm{~Pa}$; this happens since enough plasma is generated to get a $50 \Omega$ load, while at lower pressure $\left(p_{s}>0.3 \mathrm{~Pa}\right)$, reflection up to $5 \%$ can be observed.

Since the vessel gas pressure $p_{v}$ typically ranges from about $0.2 \mathrm{~Pa}$ when only two turbopumps are used to $0.02 \mathrm{~Pa}$ when one cryopump is operated, beam stripping and ionization are not negligible, and light emission (mainly Balmer emission after beam particle-gas collision) can be easily observed from television lateral cameras (TLCs) as TLC1 is toward the $x$ direction and TLC2 is

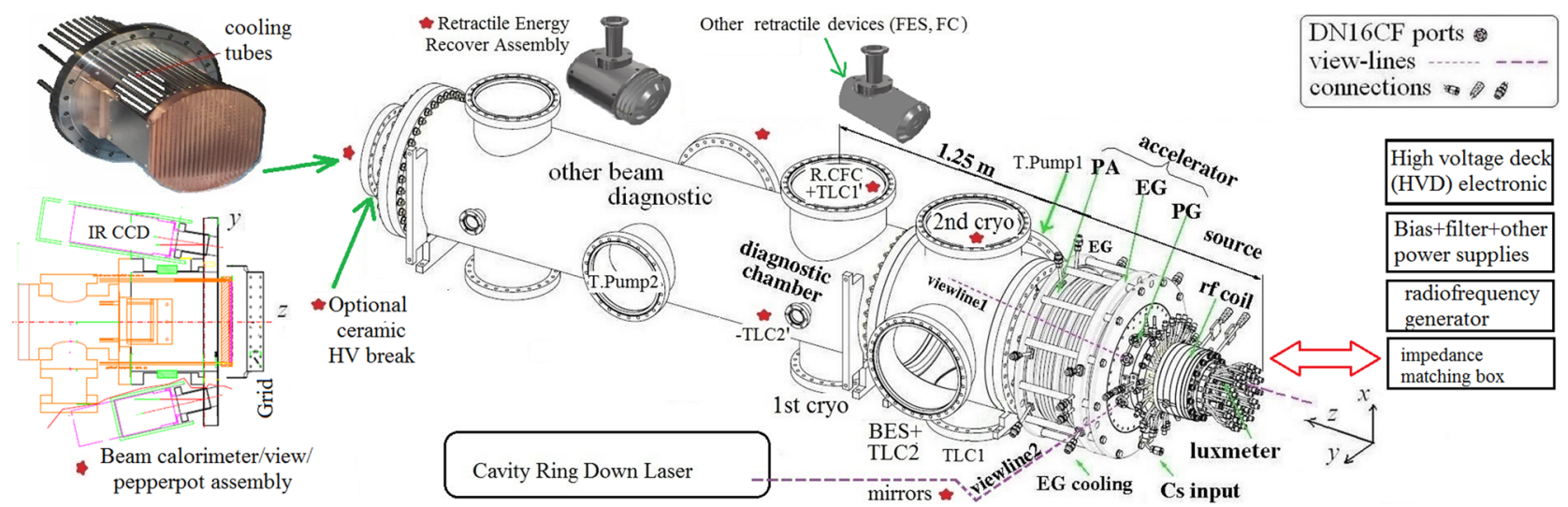

FIG. 2. NIO1 3D view including planned improvement ports (star markers), cameras (TLC), Faraday cup (FC), energy recover plugin, and end calorimeter with a possible new position of the infrared camera (IR CCD). 

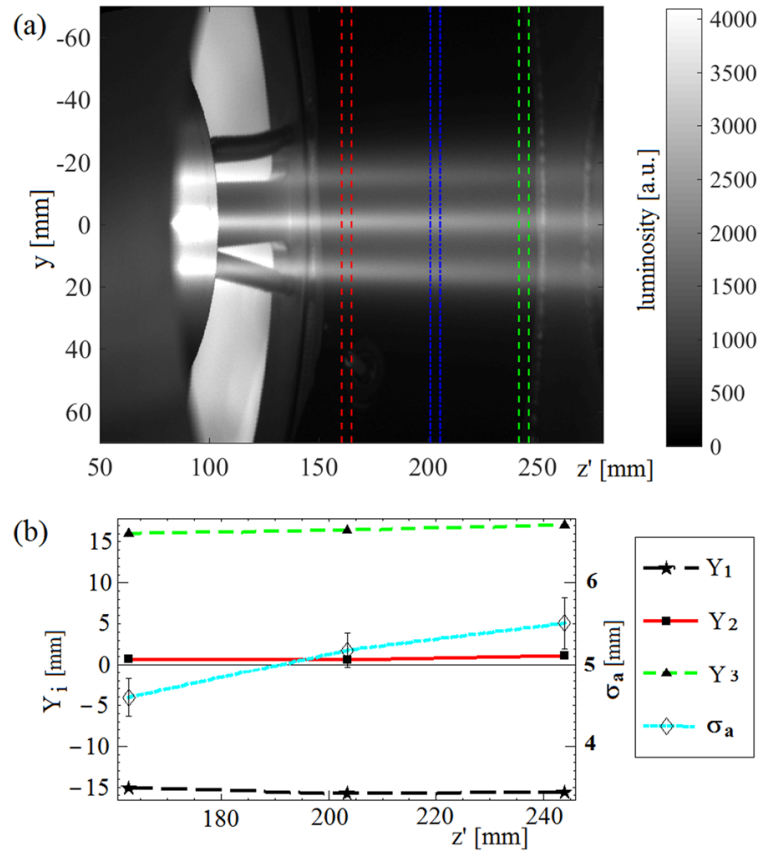

FIG. 3. (a) Image taken from monochrome camera TLC1 with 9 beamlets superposed into 3 columns (no perspective correction, coordinate refers to the median plane); note the three bands where the beam profile is sampled. (b) Fitted centroids $Y_{i}$ of the beamlet columns (typical std error $0.5 \mathrm{~mm}$, about marker size) and their rms width $\sigma_{a}$ (average of the 3 columns).

toward the $y$ direction. Beam profiles and beam divergences can thus be reconstructed ${ }^{9}$ (see Fig. 3); first, by averaging luminosity on a convenient $z$-interval, centered at $z^{\prime}=z_{1}$, a luminosity profile $L(y)$ is obtained with 3 peaks due to the columns of beamlets, where $z^{\prime}$ $=z-z_{R}$ is a local coordinate with $z^{\prime} \cong 0$ roughly being the repeller grid position; the column centroids $Y_{i}$ and rms widths $\sigma_{i}$ are obtained by a fit. The procedure is repeated for $z_{2}$ and $z_{3}$, giving the result shown in Fig. 3(b), with $\sigma_{a}$ being the average of $\sigma_{i}$. Then the widths are projected back to the repeller with linear fits,

$$
Y_{i}\left(z_{j}\right)=\alpha_{i}+\beta_{i} z_{j}, \quad \sigma_{i}\left(z_{j}\right)=\gamma_{i}+d_{i} z_{j},
$$

where the fit parameters, $\alpha_{i}, \beta_{i}, \gamma_{i}$, and the rms divergence $d_{i}$, depend on column index $i=1,2,3$. More elaborate fits will require more data and hence, in practice, more cameras to cover a larger $z$ span (we plan to put another camera TLC1 ${ }^{\prime}$ about $0.5 \mathrm{~m}$ downstream).

In the case shown, the initial centroids $\alpha_{i}$ are well compatible with the $14 \mathrm{~mm}$ spacing of grid holes, while rms divergence about $11 \mathrm{mrad}$ (averaged on 3 columns) was a promising step toward a reasonable optics and needs better optimization as discussed later.

There are some specific features of NIO1 with respect to the SPIDER, which are briefly listed in this paragraph. In NIO1, an rf coil, accelerator insulators, and a Cs heater are in air, and the HVD is at PG potential (not at the EG one); the HV power supply stops at the first breakdown, which limits CW mode operation to $V_{s}<25 \mathrm{kV}$, also depending on the pressure and $\mathrm{rf}$ power used. No rf flip is observed, and no automatic capacitance tuning is installed. There is no Faraday shield.
The most striking feature of NIO1 may be related to the rf window. It was observed, in a true Cs-free operation, that the $\mathrm{H}^{-}$current output benefits from large flows of air cooling of the rf window ( $\cong 15 \mathrm{~m}^{3} / \mathrm{h}$ at 2.5 absolute bar). Moreover, in 2019 , the $\mathrm{H}^{-}$current was increased (typically for half an order of magnitude) by one session of conditioning NIO1 with an oxygen plasma (a 5\% mixing of Ar was added for enhancing visible emission); the effect persisted for some sessions but tended to decay after 3 sessions after which the conditioning session was repeated when possible. As a practical note on the present setup, sessions include about $4 \mathrm{~h}$ of continuous beam time, as limited by scheduling and preparation time, and changing gas requires one-day stops for installing or removing the $\mathrm{O}_{2} / \mathrm{Ar}$ bottle and purging the NIO1 gas line. The installation of at least two auxiliary gas feeding points is well advanced.

Recognition of this $\mathrm{O}_{2} / \mathrm{Ar}$ effect is related to the observation ${ }^{5}$ of the inverse correlation between $I_{\mathrm{cfc}}$ and the photomultiplier signal $V_{\text {pmt }}$ (which measures plasma integrated luminosity) at constant control parameters, such as the pressure $p_{s}, \operatorname{rf}$ power $p_{k}$, and source voltages. A provisional heuristic interpretation is that a poor $I_{\mathrm{cfc}}$ is correlated with a somewhat larger electron temperature $T_{e}$ in the rf driver, which explains the larger luminosity. The effectiveness of air cooling of the rf window indicates that the window status may significantly affect driver plasma. The oxygen conditioning was suggested by previous NIO1 operations, ${ }^{8}$ where oxygen was used as the main beam, with the deposition of films on the used $\mathrm{rf}$ window (some darkening was visible). To confirm the role of the rf window, the construction of a Faraday shield plug-in for NIO1 is being considered, which seems technically challenging for its reduced size. Even if the need for a more systematic investigation is clear (which will greatly benefit from a $160 \mathrm{~mm}$ internal diameter source NIO2 being promoted), the effect might open a new perspective on Cs-free negative ion sources in the continuous regime.

The NIO1 Cs oven was designed with a heat resistant valve to protect Cs during source ventilation; for further flexibility, an $\mathrm{Ar}$ purging line was added. The first method used to test the NIO1 Cs oven prototype (in a test bench completely separated from NIO1) was deposition on square Mo targets..$^{5}$ As a second method, following the SPIDER oven experience, ${ }^{10}$ we installed a compact Surface Ionization Detector (SID) ${ }^{11}$ in that test bench. Failure of a gate valve and frequent interruptions of main power caused air leaks and oxide crusts in the Cs reservoir, which was then rebuilt [see Fig. 4(a)]. Hence, Cs oven installation in NIO1 was rescheduled in Autumn. A preliminary calibration of the SID filament current $I_{f}$ and its bias voltage $V_{1}$ measuring the emitted current $I_{1}$ was done; the filament voltage $V_{f}$ is measured by a four-wire scheme, which allows the resistance to be measured (and average filament temperature $T_{a f}$ to be inferred) with 3.5 digit precision [see Fig. 4(b)]. Dark and dispersed current contribution $I_{d}$ to $I_{1}$ changes appreciably during SID conditioning, and is not simply linear with $V_{1}$ [see Fig. 4(c)] (mainly due to the electronics used), while it does not appreciably increase with $T_{a f}$. Current $I_{f}=3.2 \mathrm{~A}$ and voltage $V_{1}=60 \mathrm{~V}$ were selected as the SID operation point, where the dark current $I_{d} \leq 90 \mathrm{nA}$. New oven operation (for $8 \mathrm{~h}$ ) up to a temperature of $130^{\circ} \mathrm{C}$ showed some slow increase in $I_{1}$ to $120 \mathrm{nA}$, with the small value being mainly explained by the comparatively large distance between oven nozzle ( $2 \mathrm{~mm}$ radius) and SID filament $(40 \mathrm{~mm})$. From a preliminary calculation of Cs flow and tabulated vapor pressure, the efficiency of the Cs transfer pipe (held at $180^{\circ} \mathrm{C}$ ) is estimated to be about $10 \%$. 

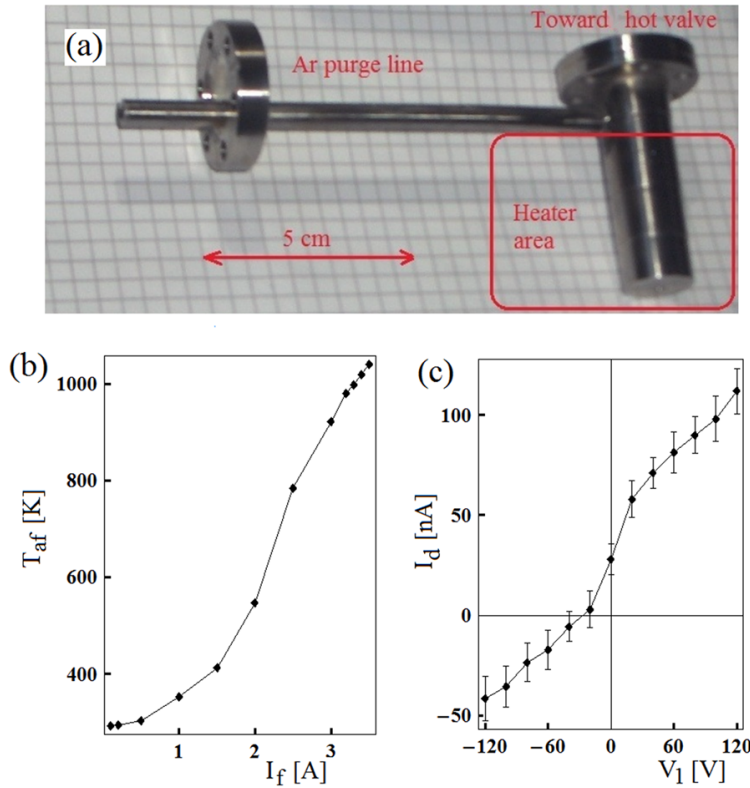

FIG. 4. (a) New Cs reservoir (with heater removed), (b) average filament temperature vs SID filament current $I_{f}$, and (c) preliminary data on dark and dispersed current $I_{d}$ vs filament bias $V_{1}$ with respect to the chamber wall.

An important NIO1 upgrade is a compact Cavity Ring Down Spectrometer to measure $\mathrm{H}^{-}$density (averaged along the line of sight), with two miniaturized shutters to protect mirrors from Cs when the laser is off; this is ready for installation on NIO1, with the exception of laser path protection cover, under construction.

The concept and plan of a beam energy recovery assembly (see Fig. 2) compatible with NIO1 are discussed elsewhere. ${ }^{12}$ Another planned upgrade is the water-cooled calorimeter (with a CF200 flange, see Fig. 2) to be placed at the diagnostic tube end. While this calorimeter has a poor spatial resolution $(7 \mathrm{~mm})$, it is suited for measuring high power beams in the CW (without side diffusion of heat). Actually, the spatial resolution corresponds to an angular resolution of $3 \mathrm{mrad}$ (and is well proportionate for a typical $10 \mathrm{mrad}$ rms divergence). Moreover, a pepperpot emittance meter is easily integrated into this detector. The calorimeter will plug the $z$-axis viewport of the infrared camera, which will be moved to an angled viewport ( $9^{\circ}$ with respect to the $z$-axis). This design also features calorimeter insulation from earth with a negatively biased suppressor grid before it; the grid may be a harp of thin wires (or a double harp) or a square mesh, whose shielding properties were calculated elsewhere. ${ }^{13}$ The suppression harp (or mesh) is a compromise between high transparency and good shielding and is convenient for Faraday Cups for large and perhaps unfocused beams.

\section{NEW FILTER FIELD AND BEAM RESULTS}

An $\mathrm{H}^{-}$source needs a magnetic field $B^{s}$ (called filter) inside the source near PG to protect fragile $\mathrm{H}^{-}$from more energetic plasma electrons; the filter optimal value and profile are still debated.

In the original NIO1 configuration, called setup "a," $B^{s}$ was aligned in the $x$ direction, to avoid interference with the deflection field $B^{d}$, and was produced only by a current $I_{\mathrm{pg}}$ limited to $400 \mathrm{~A}$; then, $B_{x}^{s}$ had a modest maximum of $4 \mathrm{mT}$ and an integral value of $\mathcal{I}_{x}=\int\left|B_{x}\right|=0.08 \mathrm{mT} \mathrm{m}$. By adding external C-shaped conductors, in 2016, we built setup "b." This gives $\mathcal{I}_{x}=0.16 \mathrm{mT} \mathrm{m}$, which was very successful in enhancing $\mathrm{O}^{-}$ion production. ${ }^{5,14}$ Let $\mathbf{B}^{(1)}$ be the field produced (by sole currents) in setup "b." Setup "c" added a permanent magnet (PM) system producing a term $\mathbf{B}^{(2)}$ with a peak $B_{y}^{(2)} \cong 15 \mathrm{mT}$, whose effectiveness was seemingly hindered by the interference with deflection field $\mathbf{B}^{d}$. In the setups " $\mathrm{d}$ " and "e," the source field $\mathbf{B}^{s}$ was left unchanged, but $\mathbf{B}^{d}$ was changed (with new extraction grid magnets labeled EG3) to obtain cancellation of ion deflection in the acceleration. ${ }^{6,15}$ In setup " $\mathrm{f}$," we replace some source magnets with a new PM system, which produces a $\mathbf{B}^{(3)}$ term with peak $B_{x}^{(3)}=-5 \mathrm{mT}$. As apparent from first experiments (end 2018), the beam was optimized by reversing $I_{\text {pg }}$ (setup "f2") so that $\mathbf{B}^{(1)}$ and $\mathbf{B}^{(3)}$ will reinforce each other (see Fig. 5). As a further test that a $\left|B_{x}\right|$ (or $\mathcal{I}_{x}$ ) increase is beneficial, we added some magnets also outside the source vacuum chamber, giving a term $B_{x}^{(4)}$ with a peak value of $-4 \mathrm{mT}$. We call this setup " $\mathrm{f3}$," where $\mathbf{B}^{s}=-\mathbf{B}^{(1)}+\mathbf{B}^{(3)}$ $+\mathbf{B}^{(4)}$. Since $B_{x}^{(1)}$ and $\mathbf{B}^{(4)}$ are easily changed without opening the ion source, the filter field $B_{x}^{s}$ now can span the $(-12,5) \mathrm{mT}$ range. As a trend, source performances improve with $\left|B_{x}\right|$, at least in NIO1 conditions (no cesiation, small radius $R_{c}$ source): $I_{\mathrm{cfc}}$ is increased and the coextracted electron current is decreased by increasing $I_{\mathrm{pg}}$ and using the "f3" setup.

In Fig. 6, results for $\left|I_{\mathrm{cfc}}\right|$ are shown, both with cryopump on (up to $7 \mathrm{~mA}$ ) and off (up to $22 \mathrm{~mA}$ ). On the other hand, it is well known that calorimetric currents $I_{\text {cal }}$ are substantially lower, since secondary plasma can contribute to $\left|I_{\mathrm{cfc}}\right|$. In the case of cryopump on, results roughly agree $\left(I_{\mathrm{cal}} /\left|I_{\mathrm{cfc}}\right| \cong 0.7\right)$ so that the ion current at extraction may reach up to $20 \mathrm{~A} / \mathrm{m}^{2}$, which is typical of volume sources. In the case of cryopump off, the difference seems larger (even one order of magnitude) so that more accurate modeling and calibration are necessary.

The $V_{s} / V_{e}$ voltage ratio was empirically adjusted (to about 9) for a better beam quality. In the case of Fig. 3, we had $V_{s}=5.4 \mathrm{kV}$ and $V_{e}=0.55 \mathrm{kV}$ with $I_{\mathrm{cfc}}=-7.64 \mathrm{~mA}$, and in the source, $p_{s}=0.9 \mathrm{~Pa}$, bias voltage $V_{b}=35 \mathrm{~V}$, and bias plate current $I_{\mathrm{bp}}=0.3 \mathrm{~A}$. In the case

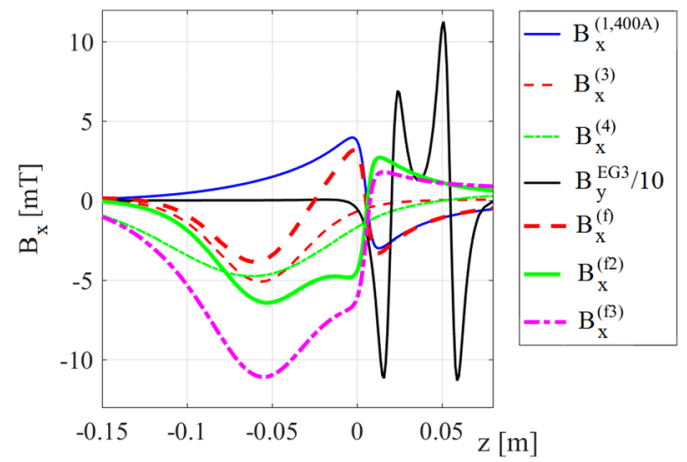

FIG. 5. Plot of $B_{x}$ vs $z$. Setups "f," " $f 2$," and "f3" are superpositions, defined in the text, of terms $B^{(1)}$ (here, at $\left.I_{\mathrm{pg}}=400 A\right), B^{(3)}$, and $B^{(4)}$. For comparison, $B_{y}$ from grid EG3 is shown (see the legend for scaling). 


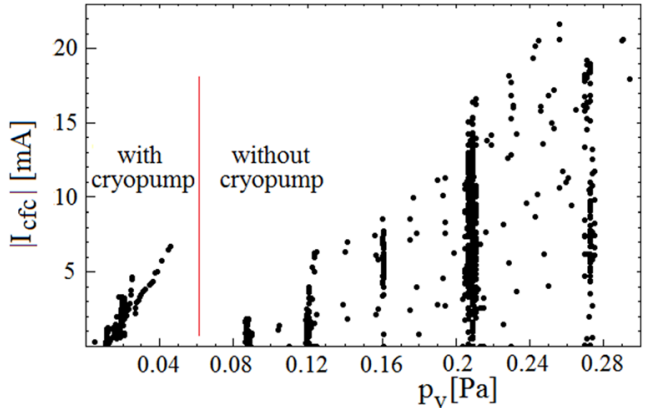

FIG. 6. Scatter plot of recent results for $\left|l_{\text {cfc }}\right|$ vs the vessel pressure $p_{v}$; setup "f3."

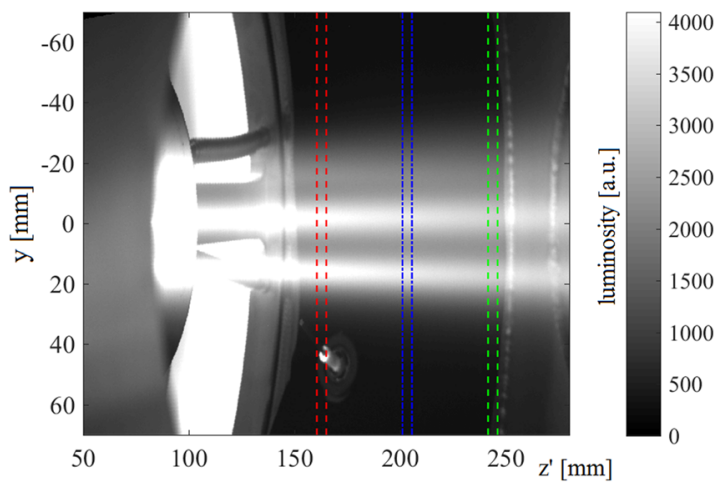

FIG. 7. Camera TLC1 with $V_{s}=11.2 \mathrm{kV}$ and $V_{e}=1.4 \mathrm{kV}$.

of Fig. 7 with $V_{s} / V_{e}=8$, the beam quality is lower also due to the larger current $\left(I_{\mathrm{cfc}}=-20.5 \mathrm{~mA}\right)$.

In Fig. 8(a) [with fixed $V_{s}=9 \mathrm{kV}$ and $V_{e}$ spanning the (0.6, 1.35) $\mathrm{kV}$ range], the central beamlet column divergence $d_{2}$ has a
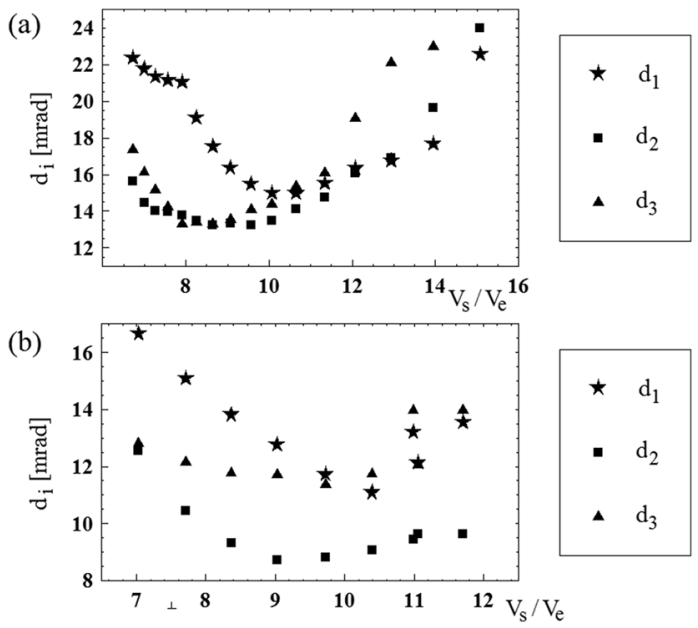

FIG. 8. (a) Beam column rms divergences $d_{i}$ vs voltage ratio $V_{e} / V_{s}$ with fixed $V_{s}=9 \mathrm{kV}$ at $p_{s}=0.75 \mathrm{~Pa}$; (b) same quantities but with fixed $V_{e}=0.6 \mathrm{kV}$ at $p_{s}=0.9 \mathrm{~Pa}$. nice (wide) minimum around $V_{s} / V_{e} \cong 9$, while lateral ones $d_{1}$ and $d_{3}$ (affected by disuniformity of the filter field ${ }^{14}$ and plasma, ${ }^{5}$ and by viewing errors) suggest using a slightly larger $V_{s} / V_{e} \cong 9.5$. In Fig. 8(b), where $V_{e}=0.6 \mathrm{kV}$ was held fixed, the $d_{2}$ optimal region (the minimum) is narrower but still around $V_{s} / V_{e} \cong 9$, and better beam optics are evident.

Since each calorimetric measurement $I_{\text {cal }}$ requires a considerable time, we plan to improve $I_{\mathrm{cfc}}$ measurement with (1) independent voltages on repeller REP, grid PA, and CFC so that a positively biased repeller collects most of secondary electrons from gas ionization; (2) a change in the CFC geometry incorporating it into a Faraday cup (FC) with a proper suppressor; (3) making CFC removable, in order to avoid beam switch on/off.

The results of setup " $\mathrm{f} 3$ " show both the advantage of operating at a larger filter field ( $B_{x} \cong 12 \mathrm{mT}$ in the NIO1 geometry) and the importance of controlling all other side variables ( $\mathrm{rf}$ window and conditioning). In conclusion, NIO1 operation has shown clear indications on the optimal filter direction and strength and on some effective beam diagnostics; of course, these are tailored to NIO1 specific conditions (CW operation, relatively small size of the plasma source). In Cs-free operation, conditioning with $\mathrm{O}_{2}$ and/or increasing rf window cooling improves the $\mathrm{H}^{-}$output. In parallel, after a commissioning of the Cs oven, some upgrading of the beam stop (water cooling) and high voltage system may be necessary for accommodating larger beam currents.

\section{ACKNOWLEDGMENTS}

Some parts of this research were financially supported by EUROFusion, commission INFN-5 (technological researches) or project INFN-E (energy).

\section{REFERENCES}

${ }^{1}$ H. Zohm, C. Angioni, E. Fable et al., Nucl. Fusion 53, 073019 (2013).

${ }^{2}$ V. Toigo, S. Dal Bello, E. Gaio et al., Nucl. Fusion 57, 086027 (2017).

${ }^{3}$ U. Fantz, P. Franzen, W. Kraus et al., Rev. Sci. Instrum. 79, 02A511 (2008).

${ }^{4}$ M. De Muri, M. Cavenago, G. Serianni et al., Fusion Eng. Des. 96, 249 (2015).

${ }^{\mathbf{5}}$ M. Cavenago, G. Serianni, C. Baltador et al., AIP Conf. Proc. 2052, 040013 (2018).

${ }^{6}$ M. Cavenago and P. Veltri, Plasma Sources Sci. Technol. 23, 065024 (2014).

${ }^{7}$ G. Serianni, M. De Muri, A. Muraro et al., Rev. Sci. Instrum. 85, 02A736 (2014).

${ }^{8}$ M. Cavenago, G. Serianni, M. De Muri et al., Rev. Sci. Instrum. 87, 02B320 (2016).

${ }^{9}$ M. Ugoletti, M. Agostini, M. Brombin, R. Pasqualotto, and G. Serianni, Europhys. Conf. Abstr. 43C, P5.3012 (2019)

${ }^{10}$ A. Rizzolo, M. Barbisan, L. Bizzotto et al., Fusion Eng. Des. 146, 676 (2019).

${ }^{11}$ M. Fröschle, R. Riedl, H. Falter, R. Gutser, U. Fantz, and IPP NNBI team, Fusion Eng. Des. 84, 788 (2009).

${ }^{12}$ V. Variale, M. Cavenago, P. Agostinetti, P. Sonato, and L. Zanotto, Rev. Sci. Instrum. 87, 02 B305 (2016).

${ }^{13}$ M. Cavenago, L. Bellan, and M. Comunian, AIP Adv. 8, 125221 (2018).

${ }^{14}$ M. Cavenago, C. Baltador, and P. Veltri, AIP Conf. Proc. 2011, 060003 (2018).

${ }^{15}$ G. Chitarin, P. Agostinetti, D. Aprile, N. Marconato, and P. Veltri, Rev. Sci. Instrum. 85, 02B317 (2014). 